\title{
An association between time-varying serum albumin level and the mortality rate in maintenance haemodialysis patients: a five- year clinical cohort study
}

Jin-Bor Chen ${ }^{1 *}$, Ben-Chung Cheng ${ }^{1}$, Cheng-Hong Yang ${ }^{2}$ and Moi-Sin Hua ${ }^{2}$

\begin{abstract}
Background: Until now, no long-term studies relating serum albumin level to mortality rate in prevalent haemodialysis (HD) patients have been conducted. We aimed to examine the association between serum albumin level and mortality over a 5-year period.

Methods: This study included 781 patients who received maintenance HD in a large, hospital-facilitated HD centre. Five-year medical records (2009-2013) were retrospectively reviewed, and the cut-off level for serum albumin level was set at $3.5 \mathrm{~g} / \mathrm{dL}$. The analysed albumin levels were expressed as time-averaged levels (first 24-month data) and albumin target reach rate over the first 2-year interval. Univariate and multivariate Cox proportional hazard regression models were used to examine the hazard function of the all-cause and cardiovascular mortality of the study participants in the subsequent 3-year period (2011-2013).

Results: Compared to those with a $100 \%$ albumin reach rate $(3.5 \mathrm{~g} / \mathrm{dL})$, the participants with $75-<100,50-<75$, and $1-<50 \%$ albumin reach rates exhibited significantly increased risk for all-cause mortality (HR 1.72, $95 \% \mathrm{Cl} 1$. 19-2.47; HR 3.14, 95 \% Cl 1.91-5.16; HR 3.66, $95 \%$ Cl 2.18-6.16, respectively). A similar trend for all-cause mortality was demonstrated in participants with time-averaged albumin levels $<4 \mathrm{~g} / \mathrm{dL}$ (HR 1.57, $95 \% \mathrm{Cl} 1.00-2.46$ for 3.5-4. $0 \mathrm{~g} / \mathrm{dL}$; HR 3.66, $95 \% \mathrm{Cl} 2.11-6.32 \mathrm{for}<3.5 \mathrm{~g} / \mathrm{dL}$ ). Compared to a $100 \%$ albumin reach rate, the $50-<75$ and $1-<$ $50 \%$ groups (HR 4.28, 95 \% Cl 1.82-10.01; HR 3.23, 95 \% Cl 1.22-8.54 respectively) showed significantly higher cardiovascular mortality rates. Similarly, participants with a time-averaged serum albumin level $<3.5 \mathrm{~g} / \mathrm{dL}$ exhibited a higher risk for cardiovascular mortality (HR 3.24, 95 \% Cl: 1.23-8.56).
\end{abstract}

Conclusions: This long-term study demonstrated that higher reach rates of serum albumin levels and higher time-averaged serum albumin levels are associated with a lower mortality rate in patients undergoing maintenance HD.

Keywords: Albumin, Mortality, Haemodialysis

\footnotetext{
* Correspondence: chenjb1019@gmail.com

${ }^{1}$ Division of Nephrology, Department of Internal Medicine, Kaohsiung Chang

Gung Memorial Hospital and Chang Gung University College of Medicine,

123 Ta Pei Rd, Niao Song District, Kaohsiung, Taiwan

Full list of author information is available at the end of the article
} 


\section{Background}

Serum albumin is commonly used as a proxy for nutritional status, as well as a marker of inflammation. A low serum albumin concentration is not only indicative of protein energy wasting in dialysis patients, but it is also a powerful predictor of the mortality risk in this population [1-4]. In addition, there is accumulating evidence that the factors causing the low albumin levels, rather than hypoalbuminaemia per se, may be associated with high mortality and morbidity in dialysis patients $[5,6]$. Several clinical conditions are associated with low serum albumin levels in dialysis patients, including infectious and inflammatory diseases [7, 8], fluid overload, inadequate dialysis [9], severe co-morbidity [10], and taste change [11]. Thus, regular monitoring of serum albumin levels is useful for predicting outcomes in dialysis patients.

In previous studies, the association between the serum albumin level and mortality in dialysis patients was based either on a baseline serum albumin level or on a mean level for the study period. One study that used time-dependent 2-year longitudinal change analysis noted that a drop in serum albumin levels during the first 6 months was associated with an increase in allcause and cardiovascular mortality in the subsequent months [2]. However, current studies are limited by their short-term nature and relatively small sample sizes.

In the present 5-year study, we investigated the percentage of each patient's serum albumin levels that reached a pre-defined target $(3.5 \mathrm{~g} / \mathrm{dL})$ during the study period. This percentage was termed the serum albumin reach rate in this study. We aimed to examine the association between time-averaged albumin and serum albumin reach rate in the first 2 -year period and the risk of mortality in the subsequent 3-year period in prevalent haemodialysis (HD) patients.

\section{Methods \\ Setting}

Patients who received regular outpatient HD (3 times per week) at Kaohsiung Chang Gung Memorial Hospital in Taiwan were included in the study. The patients were tracked from January 1, 2009 to December 31, 2013.

\section{Patients}

The records of 909 HD patients were reviewed, and 128 patients were excluded owing to incomplete demographic and laboratory data in the study period. Finally, 781 patients were considered eligible for inclusion in the survival analysis. Patient number of high flux HD were 672, high efficiency HD 109. The types of vascular access were arteriovenous fistula 731, Gore-Tex access 50. The underlying kidney diseases were renal parenchymal diseases $(n=156)$, systemic diseases $(n=392)$, obstructive nephropathy $(n=6)$, hereditary disease $(n=4)$, unknown cause $(n=221)$, poisoning $(n=2)$. During the study period, 182 patients died and 599 patients survived.

\section{Study design}

The study was a 5-year retrospective observational study. Serial haemogram results and biochemical data from the study period were collected and were analysed retrospectively. Baseline values were measured in 2009, including haemoglobin ( $\mathrm{Hb})$, albumin, blood urea nitrogen $(\mathrm{BUN})$, creatinine $(\mathrm{Cr})$, potassium $(\mathrm{K})$, corrected serum calcium $(\mathrm{Ca})$, phosphate $(\mathrm{P})$, urea reduction ratio (URR), intact parathyroid hormone, ferritin, Kt/V urea (Daugirdas method) [12], and cardiothoracic (CT) ratio. Corrected serum calcium was calculated by using the following equation: measured total $\mathrm{Ca}(\mathrm{mg} / \mathrm{dL})+0.8$ $(4.0$ - serum albumin $[\mathrm{g} / \mathrm{dL}])$. All blood samples were analysed using commercial kits and an autoanalyzer (Hitachi 7600-210, Hitachi Ltd., Tokyo, Japan). Albumin was measured using the bromocresol green method; the normal range was $3.5-5.2 \mathrm{~g} / \mathrm{dL}$. Chest radiography for the measurement of the CT ratio was performed after HD. All patients received HD with dialyzers that had an effective surface area of $>2.0 \mathrm{~m}^{2}$.

To examine the association between serum albumin levels and mortality, we used time-averaged albumin level and serum albumin reach rate to $3.5 \mathrm{~g} / \mathrm{dL}$ in the first 2 years (2009-2010) as the predictor variables and all-cause mortality and cardiovascular mortality in the subsequent 3 years (2011-2013) as outcome variables. The time-averaged albumin level was the average of the 2-year (24 months) serum albumin level. Twenty-four or less (censored) measurements of serum albumin levels were observed over the first 2 years for each patient. We defined serum albumin reach rate in the first 2 years as the percentage of albumin levels that reached $3.5 \mathrm{~g} / \mathrm{dL}$ and the percentage of albumin levels that were $\geq 3.5 \mathrm{~g} /$ dL. First, we stratified the albumin reach rate into 5 groups: $100 \%$ (all 24 monthly serum albumin levels reached $3.5 \mathrm{~g} / \mathrm{dL}), 75-<100,50-<75,1-<50$, and $0 \%$ (none of the 24 monthly serum albumin levels reached $3.5 \mathrm{~g} / \mathrm{dL})$. Second, we divided the participants into 2 groups according to the percentage of monthly serum albumin level recordings that were $\geq 3.5 \mathrm{~g} / \mathrm{dL}$ over the first 2 -year follow-up period: higher rate group ( $\geq 75 \%$ serum albumin levels were $\geq 3.5 \mathrm{~g} / \mathrm{dL}$ ) and lower rate group ( $<75 \%$ of serum albumin levels were $\geq 3.5 \mathrm{~g} / \mathrm{dL}$ ).

The protocol for the study was approved by the Committee on Human Research at Kaohsiung Chang Gung Memorial Hospital (101-1595B) for data review and was conducted in accordance with the Declaration of Helsinki. Informed consent was not required from the study patients, according to the retrospective data review regulations of the Committee on Human Research at Kaohsiung Chang Gung Memorial Hospital. 


\section{Outcome measures}

The outcome measures included the associations between serum albumin reach rates (defined as the percentage of monthly serum albumin level recordings that were $\geq 3.5 \mathrm{~g} / \mathrm{dL}$ ) and time-averaged albumin levels in the first 2-year period with all-cause and cardiovascular mortality in the subsequent 3 -year period.

\section{Statistical analysis}

All statistical analyses were carried out by using STATA (Version 11.1). For descriptive statistics, all variables were calculated as means \pm SD (standard deviation), median with interquartile ranges, and frequency and proportion of patients. For categorical variables, the differences between groups were estimated using the $\chi^{2}$ test or Fisher's exact test as appropriate. For continuous variables, the differences between groups were estimated with an independent two-sample $t$-test. In addition to descriptive statistics, both univariate and multivariate linear regression were used to analyse the association between serum albumin and baseline relevant variables. The univariate and multivariate Cox proportional hazard regression model was used to estimate the hazard function of all-cause and cardiovascular mortality for study participants, and the hazard ratios (HR), $95 \%$ confidence intervals $(95 \% \mathrm{CI})$, and $p$-values were computed. The stratified analysis for higher albumin rate and lower albumin rate participants was performed by using the Cox regression

Table 1 Baseline characteristics and clinical features of the study population with different albumin status $(n=781)$

\begin{tabular}{|c|c|c|c|c|c|}
\hline \multirow[t]{3}{*}{ Variables } & \multicolumn{4}{|c|}{ Albumin rate reached $3.5(\mathrm{~g} / \mathrm{dL})$} & \multirow[t]{3}{*}{$P$} \\
\hline & \multicolumn{2}{|c|}{ Lower rate $(n=92)$} & \multicolumn{2}{|c|}{ Higher rate $(n=689)$} & \\
\hline & Mean & SD & Mean & SD & \\
\hline Dialysis vintage (years) & 7.29 & 4.94 & 7.81 & 4.98 & 0.346 \\
\hline 5-year mortality $(n, \%)$ & & & & & $<0.001$ \\
\hline Survival & 40 & 43.48 & 559 & 81.13 & \\
\hline Cardiovascular & 18 & 19.57 & 37 & 5.37 & \\
\hline Other cause & 34 & 36.96 & 93 & 13.50 & \\
\hline Age (years) & 66.60 & 11.48 & 58.32 & 11.88 & $<0.001$ \\
\hline Gender $(n, \%)$ & & & & & 0.066 \\
\hline Male & 33 & 35.87 & 317 & 46.01 & \\
\hline Female & 59 & 64.13 & 372 & 53.99 & \\
\hline Diabetes Mellitus ( $n, \%)$ & & & & & $<0.001$ \\
\hline No & 56 & 60.87 & 540 & 78.37 & \\
\hline Yes & 36 & 39.13 & 149 & 21.63 & \\
\hline Year of death & & & & & - \\
\hline Death before 2010 & 18 & 19.57 & 19 & 2.76 & \\
\hline Death after 2010 & 34 & 36.96 & 111 & 16.11 & \\
\hline \multicolumn{6}{|l|}{ Blood analysis } \\
\hline $\mathrm{Hb}(\mathrm{g} / \mathrm{dL})$ & 10.37 & 1.68 & 10.76 & 1.23 & 0.006 \\
\hline Albumin (g/dL) & 3.43 & 0.30 & 3.95 & 0.27 & $<0.001$ \\
\hline BUN (mg/dL) & 62.67 & 18.74 & 69.26 & 16.12 & $<0.001$ \\
\hline $\mathrm{Cr}(\mathrm{mg} / \mathrm{dL})$ & 8.75 & 2.22 & 10.89 & 2.19 & $<0.001$ \\
\hline $\mathrm{K}(\mathrm{meq} / \mathrm{L})$ & 4.73 & 0.77 & 4.99 & 0.71 & $<0.001$ \\
\hline $\mathrm{Ca}(\mathrm{mg} / \mathrm{dL})$ & 9.54 & 0.81 & 9.34 & 0.85 & 0.030 \\
\hline$P(m g / d L)$ & 4.54 & 1.52 & 4.89 & 1.38 & 0.023 \\
\hline iPTH (pg/mL) & 163.45 & $(60-356)$ & 216.10 & $(76-510)$ & 0.141 \\
\hline Ferritin (ng/mL) & 443.25 & $(280-714)$ & 396.10 & $(231-595)$ & 0.022 \\
\hline $\mathrm{Kt} / \mathrm{N}$ urea & 1.69 & 0.34 & 1.74 & 0.78 & 0.540 \\
\hline URR & 0.75 & 0.07 & 0.75 & 0.07 & 0.942 \\
\hline Cardiothoracic ratio & 0.54 & 0.08 & 0.50 & 0.07 & $<0.001$ \\
\hline
\end{tabular}

$P$-value for categorical variable was estimated by $X^{2}$ test and continuous variables were estimated by two-sample $t$-test Abbreviations: URR urea reduction ratio 
Table 2 Univariate and multivariate regression analysis between baseline serum albumin levels and relevant variables

\begin{tabular}{lllll}
\hline Variable & Beta $^{\mathrm{a}}$ & $P^{\mathrm{a}}$ & Beta $^{\mathrm{b}}$ & $P^{\mathrm{b}}$ \\
\hline Dialysis vintage (years) & 0.01 & 0.709 & -0.03 & 0.41 \\
Age (years) & -0.29 & $<0.001$ & -0.17 & 0.00 \\
Gender (Female) & -0.11 & 0.002 & 0.02 & 0.61 \\
Diabetes Mellitus & -0.08 & 0.021 & 0.01 & 0.87 \\
$\mathrm{Hb}(\mathrm{g} / \mathrm{dL})$ & 0.24 & $<0.001$ & 0.18 & 0.00 \\
$\mathrm{BUN}(\mathrm{mg} / \mathrm{dL})$ & 0.20 & $<0.001$ & -0.02 & 0.57 \\
$\mathrm{Cr}(\mathrm{mg} / \mathrm{dL})$ & 0.39 & $<0.001$ & 0.29 & 0.00 \\
$\mathrm{~K}$ (meq/L) & 0.22 & $<0.001$ & 0.16 & 0.00 \\
Ca (mg/dL) & -0.09 & 0.015 & -0.03 & 0.34 \\
P (mg/dL) & 0.16 & $<0.001$ & 0.05 & 0.19 \\
iPTH (pg/mL) & 0.01 & 0.738 & -0.03 & 0.41 \\
Ferritin (ng/mL) & -0.05 & 0.162 & 0.07 & 0.04 \\
Kt/N urea & $<0.01$ & 0.946 & 0.00 & 0.91 \\
URR & -0.02 & 0.551 & 0.08 & 0.04 \\
Cardiothoracic ratio & -0.15 & $<0.001$ & -0.03 & 0.37 \\
\hline a Univariate regression analysis & & & \\
bMultivariate regression analysis & & &
\end{tabular}

model. In all analyses, a $p$-value $<0.05$ is considered statistically significant.

\section{Results}

A total of 781 patients were enrolled for the analysis. Among them, 689 patients had higher albumin reach rates and 92 patients had lower albumin reach rates over the 5-year period. Older age and higher prevalence of diabetes were identified in the patients with lower albumin reach rates. Patients with higher albumin reach rates exhibited higher survival rates than those with lower albumin reach rates. In terms of haemogram and biophysical parameters, patients with lower albumin reach rates exhibited significantly lower values for albumin, BUN, Cr, K, and P and higher levels of $\mathrm{Ca}$, ferritin, and $\mathrm{CT}$ ratio than those with higher albumin reach rates (Table 1).

The univariate and multivariate regression analysis between baseline serum albumin level and relevant variables is shown in Table 2. In the univariate regression analysis, baseline albumin level had a statistically significant inverse association with age, female sex, diabetes, Ca level, and CT ratio. For female sex participants, the predicted serum albumin levels would be $0.11 \mathrm{~g} / \mathrm{dL}$ lower than for males. Likewise, the predicted serum albumin levels of diatebes participants would be $0.08 \mathrm{~g} / \mathrm{dL}$ lower than non-diabetes participants. Moreover, baseline albumin level had a statistically significant positive association with $\mathrm{Hb}, \mathrm{BUN}, \mathrm{Cr}, \mathrm{K}$, and $\mathrm{P}$ levels. In the multivariate regression analysis, baseline albumin level had a statistically significant inverse association with age, and a positive association with $\mathrm{Hb}, \mathrm{Cr}, \mathrm{K}$, ferritin levels, and URR.

Table 3 shows the distribution of the study population in different albumin categories (using 2-year serum albumin measurement) as well as by mortality based on the different serum albumin categories. The percentage of all-cause mortality and cardiovascular mortality over the 3-year period was significantly higher with lower

Table 3 Different albumin categories using 2-year serum albumin measurement in the study population

\begin{tabular}{|c|c|c|c|c|c|c|c|c|}
\hline \multirow[t]{2}{*}{ Albumin group } & \multicolumn{2}{|l|}{ Total } & \multicolumn{3}{|c|}{ All-cause mortality } & \multicolumn{3}{|c|}{ Cardiovascular mortality } \\
\hline & $\bar{n}$ & $\%$ & $\bar{n}$ & $\%^{a}$ & $P$ & n & $\%^{\mathrm{b}}$ & $P$ \\
\hline Time-averaged serum albumin & & & & & $<0.001^{*}$ & & & $<0.001^{*}$ \\
\hline$\geq 4 \mathrm{~g} / \mathrm{dL}$ & 235 & 30.09 & 25 & 10.64 & & 8 & 3.40 & \\
\hline $3.5-<4 \mathrm{~g} / \mathrm{dL}$ & 474 & 60.69 & 115 & 24.26 & & 33 & 7.00 & \\
\hline$<3.5 \mathrm{~g} / \mathrm{dL}$ & 72 & 9.22 & 42 & 58.33 & & 14 & 19.44 & \\
\hline Albumin rate reached $3.5(\mathrm{~g} / \mathrm{dL})$ & & & & & $<0.001^{* *}$ & & & $<0.001^{* *}$ \\
\hline $100 \%$ & 487 & 62.36 & 67 & 13.76 & & 19 & 3.90 & \\
\hline 75 to $<100 \%$ & 202 & 25.86 & 63 & 31.19 & & 18 & 8.91 & \\
\hline 50 to $<75 \%$ & 46 & 5.89 & 23 & 50.00 & & 9 & 19.56 & \\
\hline 1 to $<50 \%$ & 38 & 4.87 & 24 & 63.16 & & 7 & 18.42 & \\
\hline 0 & 8 & 1.02 & 5 & 62.50 & & 2 & 25.00 & \\
\hline Albumin rate reached 3.5 (g/dL) & & & & & $<0.001^{*}$ & & & $<0.001^{*}$ \\
\hline Higher rate (75-100 \%) & 689 & 88.22 & 130 & 18.87 & & 37 & 5.37 & \\
\hline Lower rate $(0-<75 \%)$ & 92 & 11.78 & 52 & 56.52 & & 18 & 19.56 & \\
\hline
\end{tabular}

$*: X^{2}$ test

**: Fisher's exact test

${ }^{a}$ (number of all-cause mortality/total number in each category) $\times 100 \%$

$\mathrm{b}$ (number of cardiovascular mortality/total number in each category) $\times 100 \%$ 
time-averaged serum albumin level and with lower albumin reach rates over the first 2-year period.

The Cox regression survival analysis shown in Table 4 demonstrates the associations between different albumin categories (using the first 2-year serum albumin measurement) and all-cause and cardiovascular mortality over the subsequent 3-year period. The $100 \%$ albumin reach rate, higher reach rate, and time-averaged serum

Table 4 Associations between different albumin categories using 2-year serum albumin measurements with all-cause and cardiovascular mortality in the study population

\begin{tabular}{|c|c|c|c|c|c|c|c|c|c|}
\hline \multirow[t]{3}{*}{ Albumin category } & \multicolumn{9}{|c|}{ All-cause mortality } \\
\hline & \multicolumn{3}{|c|}{ Unadjusted } & \multicolumn{3}{|c|}{$\begin{array}{l}\text { Adjusted } \\
\text { (demographic) }\end{array}$} & \multicolumn{3}{|c|}{ Adjusted } \\
\hline & $\overline{\mathrm{HR}}$ & $95 \% \mathrm{Cl}$ & $P$ & $\overline{\mathrm{HR}}$ & $95 \% \mathrm{Cl}$ & $P$ & $\overline{H R}$ & $95 \% \mathrm{Cl}$ & $P$ \\
\hline \multicolumn{10}{|l|}{$\begin{array}{l}\text { Albumin rate reached } 3.5 \\
(\mathrm{~g} / \mathrm{dL})\end{array}$} \\
\hline $100 \%$ & \multicolumn{3}{|c|}{ Reference } & \multicolumn{3}{|c|}{ Reference } & \multicolumn{3}{|c|}{ Reference } \\
\hline 75 to $<100 \%$ & 2.41 & $1.70-3.43$ & $<0.001$ & 2.01 & $1.41-2.87$ & $<0.001$ & 1.72 & $1.19-2.47$ & 0.004 \\
\hline 50 to $<75 \%$ & 5.82 & $3.61-9.37$ & $<0.001$ & 3.64 & $2.22-5.95$ & $<0.001$ & 3.14 & $1.91-5.16$ & $<0.001$ \\
\hline 1 to $<50 \%$ & 7.20 & $4.43-11.69$ & $<0.001$ & 4.95 & $3.00-8.16$ & $<0.001$ & 3.66 & $2.18-6.16$ & $<0.001$ \\
\hline 0 & 8.10 & $3.25-20.14$ & $<0.001$ & 4.80 & $1.90-12.15$ & 0.001 & 2.54 & $0.96-6.76$ & 0.062 \\
\hline \multicolumn{10}{|l|}{$\begin{array}{l}\text { Albumin rate reached } 3.5 \\
(\mathrm{~g} / \mathrm{dL})\end{array}$} \\
\hline Higher rate (75-100 \%) & \multicolumn{3}{|c|}{ Reference } & \multicolumn{3}{|c|}{ Reference } & \multicolumn{3}{|c|}{ Reference } \\
\hline Lower rate $(0-<75 \%)$ & 4.45 & $3.22-6.14$ & $<0.001$ & 3.11 & $2.23-4.35$ & $<0.001$ & 2.55 & $1.79-3.63$ & $<0.001$ \\
\hline \multicolumn{10}{|l|}{$\begin{array}{l}\text { Time-averaged serum } \\
\text { albumin }\end{array}$} \\
\hline$\geq 4$ & \multicolumn{3}{|c|}{ Reference } & \multicolumn{3}{|c|}{ Reference } & \multicolumn{3}{|c|}{ Reference } \\
\hline 3.5 to $<4$ & 2.38 & $1.54-3.68$ & $<0.001$ & 1.80 & $1.15-2.81$ & 0.010 & 1.57 & $1.00-2.46$ & 0.051 \\
\hline$<3.5$ & 8.71 & $5.28-14.37$ & $<0.001$ & 4.84 & $2.84-8.26$ & $<0.001$ & 3.66 & $2.11-6.32$ & $<0.001$ \\
\hline \multicolumn{10}{|l|}{$\begin{array}{l}\text { Albumin rate reached } 3.5 \\
(\mathrm{~g} / \mathrm{dL})\end{array}$} \\
\hline $100 \%$ & \multicolumn{3}{|c|}{ Reference } & \multicolumn{3}{|c|}{ Reference } & \multicolumn{3}{|c|}{ Reference } \\
\hline 75 to $<100 \%$ & 2.32 & $1.18-4.54$ & 0.015 & 1.99 & $1.01-3.93$ & 0.046 & 1.73 & $0.86-3.48$ & 0.124 \\
\hline 50 to $<75 \%$ & 7.88 & $3.53-17.58$ & $<0.001$ & 4.78 & 2.09-10.96 & $<0.001$ & 4.28 & $1.82-10.01$ & 0.001 \\
\hline 1 to $<50 \%$ & 7.75 & $3.23-18.62$ & $<0.001$ & 4.69 & $1.91-11.49$ & 0.001 & 3.23 & $1.22-8.54$ & 0.018 \\
\hline 0 & 10.38 & $2.40-44.91$ & 0.002 & 6.59 & $1.47-29.61$ & 0.014 & 4.08 & $0.86-19.39$ & 0.077 \\
\hline \multirow[t]{4}{*}{ Albumin category } & \multicolumn{9}{|c|}{ Cardiovascular mortality } \\
\hline & \multirow{2}{*}{\multicolumn{3}{|c|}{ Unadjusted }} & \multirow{2}{*}{\multicolumn{3}{|c|}{$\begin{array}{l}\text { Adjusted } \\
\text { (demographic) }\end{array}$}} & \multirow{2}{*}{\multicolumn{3}{|c|}{ Adjusted }} \\
\hline & & & & & & & & & \\
\hline & $\mathrm{HR}$ & $95 \% \mathrm{Cl}$ & $P$ & $H R$ & $95 \% \mathrm{Cl}$ & $P$ & $H R$ & $95 \% \mathrm{Cl}$ & $P$ \\
\hline \multicolumn{10}{|l|}{$\begin{array}{l}\text { Albumin rate reached } 3.5 \\
(\mathrm{~g} / \mathrm{dL})\end{array}$} \\
\hline Higher rate (75-100\%) & \multicolumn{3}{|c|}{ Reference } & Refer & & & Refer & & \\
\hline Lower rate $(0-<75 \%)$ & 5.88 & $3.31-10.45$ & $<0.001$ & 3.66 & $2.01-6.68$ & $<0.001$ & 2.94 & $1.58-5.47$ & 0.001 \\
\hline $\begin{array}{l}\text { Time-averaged serum } \\
\text { albumin }\end{array}$ & & & & & & & & & \\
\hline$\geq 4$ & Refere & & & Refer & & & Refer & & \\
\hline 3.5 to $<4$ & 2.02 & $0.93-4.42$ & 0.076 & 1.51 & $0.68-3.36$ & 0.315 & 1.28 & $0.57-2.90$ & 0.552 \\
\hline$<3.5$ & 8.94 & $3.74-21.37$ & $<0.001$ & 4.61 & $1.82-11.63$ & 0.001 & 3.24 & $1.23-8.56$ & 0.018 \\
\hline
\end{tabular}

Univariate and multivariate Cox proportional hazard regression model

Adjusted (demographic), the adjusted covariates including dialysis vintage, age, sex, and diabetes mellitus

Adjusted, the adjusted covariates including dialysis vintage, age, sex, diabetes mellitus, and all laboratory blood analysis values 
albumin $\geq 4$ were set as the references. Compared to a $100 \%$ albumin reach rate the $75-<100,50-<75$, and $1-<50 \%$ albumin reach rates exhibited significant increased risk in all-cause mortality according to the total adjusted analysis (HR 1.72, 95 \% CI 1.19-2.47; HR 3.14, 95 \% CI 1.91-5.16; and HR 3.66, 95 \% CI 2.18-6.16, respectively) A similar trend in all-cause mortality was demonstrated with higher rate albumin reach (75-100\%) and higher time-averaged albumin levels. Compared to a $100 \%$ albumin reach rate, $50-<75$ and $1-<50 \%$ reach rates were associated with significantly higher cardiovascular mortality (HR 4.28, 95 \% CI 1.82-10.01 and HR 3.23, $95 \%$ CI 1.22-8.54, respectively). Similarly, time-averaged serum albumin levels $<3.5 \mathrm{~g} / \mathrm{dL}$ conferred higher risk of cardiovascular mortality (HR 3.24, 95 \% CI: 1.23-8.56) (Table 4).

\section{Discussion}

Serum albumin level is recognized as a strong predictor of mortality in HD patients [1-4]. However, serum albumin levels in routine blood tests are known to undergo dynamic changes. Therefore, a majority of clinical studies have used baseline levels, mean levels during a shortterm period, or time-varying albumin levels to predict the risk of mortality in HD patients [1-4]. The present study investigated the association of 2-year serum albumin levels with mortality in a longitudinal 3-year period by utilizing the serum albumin reach rate for a predefined target level and the time-averaged albumin level. The cut-off serum albumin level was set at $3.5 \mathrm{~g} / \mathrm{dL}$, based on the lower normality limit in our laboratory test and the minimal requirement of the nutritional quality by the National Health Insurance Administration in Taiwan (http://sc-dr.tw/news/104/010603.htm) (http://sc-dr.tw/news/104/01/01060301.pdf). The study demonstrated that HD patients who reached the target serum albumin levels more frequently and who had higher time-averaged albumin levels over a 2-year period had a survival advantage in the subsequent 3-year period.

In this study, the baseline serum albumin level was found to be inversely associated with age, female sex, diabetes, $\mathrm{Ca}$ level, and CT ratio. Furthermore, the baseline serum albumin was found to be positively associated with, $\mathrm{Hb}, \mathrm{BUN}, \mathrm{Cr}, \mathrm{K}$, and $\mathrm{P}$ levels through univariate regression analysis. However, age was the only factor found to be inversely associated by multivariate regression analysis. A plausible explanation is that age is associated with a greater risk of disability and as well as limitation of oral intake. These conditions can result in protein malnutrition and a low albumin level.

Multivariate regression analysis showed that baseline serum albumin level was positively associated with $\mathrm{Hb}$, $\mathrm{Cr}, \mathrm{K}$, ferritin levels, and URR. This is consistent with a previous study on a cohort of HD patients, which showed the association of 3-month average serum albumin levels with $\mathrm{Hb}$ and $\mathrm{Cr}$ levels [2]. It is reasonable to assume that patients with higher $\mathrm{Hb}$ levels may have greater activity levels and thus higher dietary intake. Furthermore, a higher serum $\mathrm{Cr}$ level potentially reflects a higher muscle mass and indicates a healthy nutritional status. It is also reasonable to assume that free access to dietary intake may result in a positive association of serum albumin level with serum $\mathrm{K}$ levels.

The adequacy of dialysis is critical for predicting morbidity and mortality among HD patients. One retrospective study of approximately 10,000 HD patients found that lower urea reduction ratios during dialysis were associated with increased risk of death; the risk was increased owing to inadequate nutrition [9]. Another study of $1600 \mathrm{HD}$ patients concluded that, after reaching adequacy targets (Kt/V urea, URR), the main determinants of survival were nutrition status and age [13]. Our participants had adequate $\mathrm{Kt} / \mathrm{V}$ urea scores, and a majority had adequate URR. Furthermore, a positive association was observed between URR and the baseline serum albumin level. Accordingly, our results are consistent with the previous findings that URR and serum albumin level may contribute to the risk of death in HD patients.

Previous studies have reported the association of serum albumin levels with mortality in dialysis patients $[1-4,7,10]$. However, most of the studies had shortterm observational designs with durations between 1 and 2 years. Moreover, the outcome measures in prior studies were the baseline albumin level or the time-varying/average albumin level during the study period. In an earlier study with a follow-up of 1.25 years, baseline serum albumin level was found to be a strong predictor of death in chronic HD patients [1]. Another study with a 10-year observation period also showed that reduced baseline serum albumin level was an independent predictor of mortality in incident dialysis patients [10]. One study used trimonthly-varying serum albumin level and baseline albumin level to predict the mortality in HD patients over a 2year follow-up period. The study demonstrated that the hazard ratios for cardiovascular death increase across decrements of baseline serum albumin levels; however, the hazard ratios of cardiovascular death did not differ with time-varying albumin levels $<3.8 \mathrm{~g} / \mathrm{dL}$ [2].

The present study is the first to utilize the serum albumin reach rate in prevalent HD patients. In addition, this study had a longer observational period than previous studies. We found that higher albumin reach rate and time-averaged serum albumin level over a 2-year period are associated with lower all-cause mortality over a 3-year follow-up period. A similar trend was also observed in association with cardiovascular mortality.

While most studies have investigated the association of serum albumin levels with all-cause mortality or 
cardiovascular mortality, one study conducted in Canadian HD patients found that the serum albumin levels were associated with septicaemia and hospitalization in patients with infectious diseases [7]. In the present study, we did not determine the exact cause of mortality; therefore, the weight contribution of septicaemia in all-cause mortality was not ascertained. Taken together, the results of the previous studies and that of our present study were consistent with regards to the association between serum albumin levels and mortality in dialysis patients, with either time-averaged albumin levels or albumin reach rate used as predictors.

Although the present study showed a significant association between low serum albumin levels and mortality in prevalent HD patients, chance findings cannot be completely excluded. Retrospectively selecting participants from one large HD centre may have influenced the results. Therefore, the association between the predictor variables and mortality may be overestimated. Furthermore, the present study did not investigate the contributions of inflammation, comorbidities, and nutritional status. Finally, we used albumin reach percentage in the first 2 years and time-averaged albumin level to predict mortality in the subsequent 3 years in our HD patients. However, there still exists a drawback for fully evaluation the exposure (albumin level) and mortality in our HD patients. A well-designed, prospective study is needed to overcome the drawback in retrospective study in the future.

\section{Conclusions}

The present study indicates that a higher serum albumin level has a survival benefit in long-term HD patients. An obviously low albumin level should emphasize the need for initiating a management strategy and receiving appropriate medical intervention.

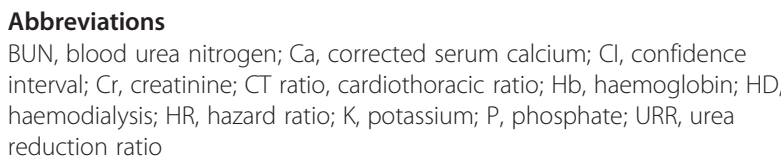

\section{Acknowledgements}

The authors thank Miss Ching-Yi Yu and Li-Chueh Kuo for their assistance in data management.

\section{Funding sources}

No funding was received for writing this study.

\section{Availability of data and materials}

All data supporting the study is presented in the manuscript or available upon request from the corresponding authos of this manuscript, Jin-Bor Chen.

\section{Authors' contributions}

JBC and BCC collected and interpreted the data and critically reviewed manuscripts. JBC drafted the manuscript. MSH performed statistical analysis. $\mathrm{CHY}$ critically reviewed the statistical data. All authors read and approved the final manuscript.

\section{Competing interests}

The authors declare that they have no competing interests.

\section{Consent for publication}

Not applicable.

\section{Ethics approval and consent to participate}

The protocol for the study was approved by the Committee on Human Research at Kaohsiung Chang Gung Memorial Hospital (101-1595B) for retrospective medical data review. Informed consent was not required from the study patients, according to the retrospective data review regulations of the Committee on Human Research at Kaohsiung Chang Gung Memorial Hospital.

\section{Author details}

${ }^{1}$ Division of Nephrology, Department of Internal Medicine, Kaohsiung Chang Gung Memorial Hospital and Chang Gung University College of Medicine,

123 Ta Pei Rd, Niao Song District, Kaohsiung, Taiwan. ${ }^{2}$ Department of Electronic Engineering, National Kaohsiung University of Applied Sciences, Kaohsiung, Taiwan.

Received: 8 May 2016 Accepted: 7 August 2016

Published online: 20 August 2016

\section{References}

1. Iseki K, Kawazoe N, Fukiyama K. Serum albumin is a strong predictor of death in chronic dialysis patients. Kidney Int. 1993;44:115-9.

2. Kalantar-Zadeh K, Kilpatrick RD, Kuwae N, McAllister CJ, Alcom Jr H, Kopple $J \mathrm{D}$, et al. Revisiting mortality predictability of serum albumin in the dialysis population: time dependency, longitudinal changes and populationattributable fraction. Nephrol Dial Transplant. 2005;20:1880-8.

3. Amaral S, Hwang W, Fivush B, Neu A, Frankenfield D, Furth S. Serum albumin level and risk for mortality and hospitalization in adolescents on hemodialysis. Clin J Am Soc Nephrol. 2008;3:759-67.

4. de Mutsert R, Grootendorst DC, Indemans F, Boeschoten EW, Krediet RT, Dekker FW. Association between serum albumin and mortality in dialysis patients is partly explained by inflammation, and not by malnutrition. J Ren Nutr. 2009;19:127-35.

5. Yeun JY, Kaysen GA. Factors influencing serum albumin in dialysis patients. Am J Kidney Dis. 1998:32:S118-25.

6. Don BR, Kaysen G. Serum albumin: relationship to inflammation and nutrition. Semin Dial. 2004;17:432-7.

7. Churchill DN, Taylor DW, Cook RJ, Laplante P, Barre P, Cartler P, et al. Canadian hemodialysis morbidity study. Am J Kidney Dis. 1992;19:214-34.

8. Kaysen GA, Stevenson FT, Depner TA. Determinants of albumin concentration in hemodialysis patients. Am J Kidney Dis. 1997;29:658-68.

9. Owen Jr WF, Lew NL, Liu Y, Lowrie EG, Lazarus JM. The urea reduction ratio and serum albumin concentration as predictors of mortality in patients undergoing hemodialysis. N Engl J Med. 1993;329:1001-6.

10. Chan M, Kelly J, Batterham M, Tapsell L. Malnutrition (subjective global assessment) scores and serum albumin levels, but not body mass index values, at initiation of dialysis are independent predictors of mortality: a 10-year clinical cohort study. J Ren Nutr. 2012;22:547.

11. Steiber AL. Clinical indicators associated with poor oral intake of patients with chronic renal failure. J Ren Nutr. 1999;9:8488.

12. Daugirdas JT. The post: pre-dialysis plasma urea nitrogen ratio to estimate $K$. t/N and NPCR: mathematical modeling. Int J Artif Organs. 1989;12:411-9.

13. Combe C, Chauveau P, Laville M, Fouque D, Azar R, Cano N, et al. Influence of nutritional factors and hemodialysis adequacy on the survival of 1,610 French patients. Am J Kidney Dis. 2001;37:S81-8. 